\title{
Composición, Análisis Termofísico y Análisis Sensorial de Frutos Colombianos. Parte 1: Almendro (Terminalia Catappa L.)
}

\author{
Guillermo Arrázola(1), Manuel Páez ${ }^{(2)}$ y Armando Alvis ${ }^{(2)}$ \\ (1) Universidad de Córdoba, Programa de Ingeniería de Alimentos, Facultad de Ingenierías. Grupo de \\ Investigación Procesos y Agroindustria de vegetales. Carrera 6 No76-103, Km 3, vía Cereté. Córdoba- \\ Colombia. email: (e-mail: guillermo.arrazola@ua.es) \\ (2) Universidad de Córdoba. Programa de Química. Facultad de Ciencias básicas. Km 3 Vía Cereté. \\ Córdoba-Colombia.
}

Recibido Oct. 14, 2013; Aceptado Dic. 13, 2013; Versión final recibida Dic. 18, 2013

\begin{abstract}
Resumen
Se determinó los contenidos de grasa, humedad y cenizas en almendras producidas en el departamento de Córdoba en Colombia. Se estudió también las propiedades termofísicas y sensoriales de la almendra fresca y tostada. Las propiedades termofísicas, como conductividad, difusividad térmica, calor específico y densidad, fueron determinadas en el fruto de acuerdo a la composición bromatológica de la almendra. Las semillas del fruto fueron tostadas a temperaturas de 110 y $140 \stackrel{\circ}{C}$ por 30 min y sometidas a evaluación sensorial. Los valores cenizas y contenido de humedad en semillas, no mostraron diferencias significativas en las regiones del alto y medio Sinú y el contenido de grasa presentó diferencias significativas $(p<0,05)$ para las zonas en estudio. La difusividad, conductividad térmica, calor especifico y la densidad de almendras frescas fue de $1.13 \times 10^{-7} \mathrm{~m}^{2} \mathrm{~s}^{-1}, 0.32 \mathrm{~W} \mathrm{~m} \mathrm{~m}^{-1} \mathrm{C}^{-1}, 2.65 \mathrm{~kJ} \mathrm{~kg}^{-1}{ }^{\circ} \mathrm{C}^{-1}$ y $1138.6 \mathrm{~kg} \mathrm{~m}^{-3}$ respectivamente. La evaluación sensorial de almendras tostadas mostró que hay diferencia entre las almendras tostadas a $140^{\circ} \mathrm{C}$ y a $110^{\circ} \mathrm{C}$, existiendo un mayor grado de satisfacción por la almendra tostada a $140^{\circ} \mathrm{C}$.
\end{abstract}

Palabras clave: almendras, propiedades termofísicas, frutos tostados, análisis sensorial

\section{Composition, Thermophysical Analysis and Sensory Analysis of Colombian Fruits. Part 1: Almond (Terminalia Catappa L.)}

\begin{abstract}
Fat contents, moisture and ashes contained in almonds produced in the department of Córdoba in Colombia were determined. Also the thermophysical and sensory properties of fresh and toasted almond were studied. The thermophysical properties, such as conductivity, thermal diffusivity, specific heat and density were determined in the fruits according to the bromatological composition of the almond. The fruit seeds were roasted at temperatures of 110 and $140{ }^{\circ} \mathrm{C}$ for $30 \mathrm{~min}$ and subjected to sensory evaluation. Ash values and moisture content in seeds showed no significant differences in the regions of high and medium Sinu and fat content showed significant differences $(p<0.05)$ for the areas under study. The diffusivity, thermal conductivity, specific heat and density of fresh almonds was $1.13 \times 10-7 \mathrm{~m}^{2} \mathrm{~s}^{-1}, 0.32 \mathrm{~W} \mathrm{~m}^{-1} \mathrm{C}^{-1}, 2.65$ $\mathrm{kJ} \mathrm{kg}^{-1}{ }^{\circ} \mathrm{C}^{-1}$ and $1138.6 \mathrm{~kg} \mathrm{~m}^{-3}$ respectively. The sensory evaluation of roasted almonds showed no difference between the toasted almonds at $140{ }^{\circ} \mathrm{C}$ and at $110^{\circ} \mathrm{C}$, but the level of satisfaction was greater for the almonds toasted at $140^{\circ} \mathrm{C}$.
\end{abstract}

Keywords: almonds, thermophysical properties, roasted fruits, sensory analysis 


\section{INTRODUCCIÓN}

El almendro de la India es un árbol grande naturalmente extendido en las zonas tropicales y subtropicales de la India y el océano pacifico y es plantado en todo el trópico (Thomson y Evans, 2006). La almendra de la India, es un fruto comprimido lateralmente, de forma ovoides a ovadas y con drupa de piel lisa. Durante la maduración, cambia del color verde a amarillo o a rojo brillante en plena madurez. El fruto se compone de núcleo $(10,32 \%)$, cubierta fibrosa $(8,97 \%)$, cáscara $(34,08 \%)$ y endocarpio duro $(46,63 \%)$ (Inbaraj y Sulochana 2006).

Hoy día, los frutos secos son un componente saludable en la dieta, por su elevado valor nutritivo y sus apreciadas características organolépticas. Los beneficios en el consumo se encuentran asociados en gran parte al perfil nutricional de la almendra, en particular a sus contenidos en tocoferol, ácidos oleico, fitoesteroles y compuestos fenólicos (Chen y Blumberg, 2008). Se han realizado numerosas investigaciones con el fruto del almendro de la India, sin embargo éstas han sido orientadas al análisis de sus propiedades medicinales y farmacológicas (Nagappa et al., 2003). De esta manera son escasas las investigaciones cuyo interés sea el aprovechamiento nutricional e industrial de la almendra proveniente del fruto y en el conocimiento de sus propiedades termofísicas.

Las propiedades termofísicas son parámetros importantes en la descripción de la transferencia de calor durante el calentamiento de alimentos sólidos, suscitando grandes ventajas en los costos energéticos, la calidad y la seguridad del alimento (Feyissa, 2011; Hassan y Ramaswamy 2011). El calor específico Cp, difusividad térmica $\alpha$ y conductividad térmica $k$; son las propiedades termofísicas más importantes en el procesamiento de alimentos (Bitra et al., 2010; Feyissa 2011). El objetivo del presente trabajo fue analizar las propiedades termofísicas y sensoriales de la semilla tostadas de almendras (Terminalia catappa L) y el estudio de contenidos de grasa,cenizas y humedad de almendras.

\section{MATERIALES Y MÉTODOS}

\section{Recolección de almendras}

Los frutos del almendro de la India (Terminalia catappa) fueron recolectados al azar, en árboles existentes en las tres subregiones del Alto, Medio y Bajo Sinú del departamento de Córdoba (Colombia); en la zona del Alto Sinú el área de estudio comprendió el municipio de Valencia, en la zona del Medio Sinú se realizó la recolección en los municipios de Cereté y San Carlos y en la zona del Bajo Sinú se realizaron recolecciones en el municipio de Lorica. En cada región del Alto, Medio y Bajo Sinú se muestrearon 25 árboles aproximadamente, recolectándose 20 almendras maduras de cada árbol.

\section{Caracterización bromatológica de la semilla del almendro de la India.}

Se realizó la extracción manual de la semilla. La caracterización de las nueces se realizó mediante la utilización de las técnicas del análisis proximal, Humedad: Método 930.15/90 AOAC; Determinación Extracto Etéreo: Método 920.39/90 AOAC; Determinación Cenizas: Método 942.04/90 AOAC; Determinación Fibra cruda: Método 962.09/90.AOAC; Determinación Proteína Cruda 955.04/90 AOAC. Determinación de grasa: Método NTC 336.2002.

\section{Análisis Termofísico de la semilla del almendro de la India.}

Las propiedades termofísicas, como conductividad térmica, la difusividad térmica, el calor específico y la densidad, se determinaron de acuerdo a la composición bromatológica de la almendra, empleando los modelos mostrados en la Tabla 1. El modelo matemático más utilizado para conocer las propiedades termofísicas es el desarrollado por Choi y Okos (1985) basado en la temperatura, en un rango de - 40ㄷ a $150^{\circ} \mathrm{C}$, y la composición que tiene el alimento de humedad, proteína, grasa, fibra, carbohidratos y cenizas y se comparó con el software elaborado por Alvis et al. (2012) para determinar propiedades termofísicas.

\section{Tostado de almendras}

Las semillas del fruto del almendro enteras y sin daños mecánicos fueron tostadas en un horno $(E \& Q$; Electrónica \& química). Para el proceso de tostado se emplearon temperaturas de 110 y $140 \stackrel{\circ}{ } \mathrm{C}$ por un tiempo de $30 \mathrm{~min}$, las cuales son los rangos de temperatura y tiempo comúnmente empleados por la industria de almendras. Se realizó un análisis sensorial a las almendras tostadas, empleando la prueba de medición del grado de satisfacción, en esta prueba afectiva se contó con 50 panelistas no entrenados compradores del tipo de alimento en cuestión, cuyas edades estaban comprendidas entre los 16 y 50 años de edad y posibles consumidores. Se evaluó la aceptación o rechazo del producto empleando como instrumento de medición una escala hedónica verbal de 5 puntos. 
Tabla 1. Modelos utilizados para la determinación de propiedades termofísicas

\begin{tabular}{lc} 
Conductividad Térmica $(k)$ & $K=\sum k_{i} X_{i}^{v}$ \\
Densidad $(\rho)$ & $\rho=\frac{1}{\sum X_{i}^{w} / \rho_{i}}$ \\
Difusividad Térmica $(\alpha)$ & $\alpha=\sum \alpha_{i} X_{i}^{v}$ \\
Calor Específico $\left(C_{p}\right)$ & $C_{p}=\sum C p_{i} X_{i}^{w}$ \\
\hline
\end{tabular}

En la Tabla, $X_{i}^{w}$ es la fracción peso del componente i, $\rho_{i}$ es la densidad del componente i puro (proteína, grasa) y $X_{i}^{v}$ es la fracción volumen estimada para el componente i (Adaptada de Choi y Okos, 1985)

\section{Análisis estadístico}

El diseño de la investigación fue un diseño experimental completamente al azar con tres repeticiones por tratamiento, bajo una estructura de tratamiento unifactorial. Las variables independientes fueron las regiones del Alto, Medio y Bajo Sinú y las variables dependientes la constituyeron los porcentajes de humedad, cenizas y grasa. Para cada variable se llevó a cabo un análisis de varianza ANOVA y se utilizó la prueba de Tukey para determinar las diferencias significativas entre las tres regiones estudiadas. En la elaboración de almendras tostadas, según los resultados obtenidos en las pruebas de aceptación, se realizó una prueba de Friedman y Anava con comparación de medias de Tukey.

\section{RESULTADOS Y DISCUSIÓN}

Análisis de Contenido de Grasa, Humedad y Cenizas de la Semilla del Almendro.

Los contenidos de grasa, humedad y cenizas de la semilla del almendro de la India para las tres regiones estudiadas se detallan en la Tabla 2. El contenido promedio porcentual de grasas en la semilla de la almendra, de las tres regiones alto, medio y bajo Sinú mostraron diferencias significativas $(p<0,05)$, Siendo el mayor porcentaje de grasa obtenido en la zona del medio Sinú con un valor del $60.3 \%$ (Tabla 2). González et al. (2005) y Buelvas et al. (2007) obtuvieron valores muy similares.

Tabla 2. Análisis composicional de almendras en tres regiones

\begin{tabular}{cccc}
\hline Composicion & Bajo Sinu & Medio Sinu & Alto Sinu \\
\hline Grasa & $56.12 \mathrm{a}$ & $60.31 \mathrm{~b}$ & $54.56 \mathrm{c}$ \\
Humedad & $22.40 \mathrm{a}$ & $27.46 \mathrm{~b}$ & $27.98 \mathrm{~b}$ \\
Cenizas & $5.23 \mathrm{a}$ & $4.91 \mathrm{~b}$ & $5.04 \mathrm{~b}$ \\
\hline
\end{tabular}

En la Tabla 2, los resultados son en base seca y las letras diferentes muestran diferencias estadísticamente significativa En cuanto a los valores de humedad y ceniza no se observaron diferencias estadísticamente significativas en las frutas provenientes de las zonas del medio y el alto Sinú, sin embargo se observó diferencias estadísticamente significativas $(p<0.05)$ entre la región alto y medio Sinú con respecto a región del bajo Sinú. Los porcentajes más altos de humedad fueron los de las frutas provenientes de la región del alto Sinú.

Las diferencias obtenidas en los contenidos de grasa, ceniza y humedad para las tres zonas en estudio denotan influencia significativa de las condiciones de los suelos, la temperatura, la pluviosidad, la altitud, la textura del suelo, minerales, oligoelementos y otros nutrientes que se encuentra en cada una de las regiones. Los contenidos de grasa y humedad son factores importantes en el tratamiento de las semillas potencialmente destinadas al procesamiento industrial, debido a que son parámetros importantes en la conservación de la materia prima y además juegan un papel importante en el aprovechamiento tecnológico de la almendra. Asimismo el alto porcentaje en grasa cruda, ofrece la oportunidad de extraer el aceite, con la finalidad de aprovecharlo en la elaboración de productos farmacéuticos y cosméticos. Los frutos secos son un componente saludable en la dieta, por su elevado valor nutritivo y sus apreciadas características organolépticas. Los beneficios en el consumo se encuentran asociados en gran parte al perfil nutricional de la almendra, en particular a sus contenidos en tocoferol, ácidos oleico, fitoesteroles y compuestos fenólicos (Chen y Blumberg, 2008). 


\section{Análisis termofísico de almendras frescas y tostadas}

Las propiedades termofísicas del almendro se calcularon a partir de la composición de la almendra (Tabla 3) utilizando las ecuaciones de la Tabla 1. Los resultados mostrados en la tabla son en base húmeda.

Tabla 3. Composición de almendra fresca.

\begin{tabular}{cc}
\hline Composicion & Almendra Fresca* \% \\
\hline Humedad & 32,71 \\
Cenizas & 5,82 \\
Grasa & 34,5 \\
Proteína & 14,82 \\
Fibra & 10,37 \\
Carbohidratos & 1,78 \\
\hline
\end{tabular}

Tabla 4. Propiedades Termofísicas

\begin{tabular}{ccccc}
\hline Propiedad & $k\left(\mathrm{~W} \mathrm{~m}^{-1} \mathrm{C}^{-1}\right)$ & $C_{p}\left(\mathrm{KJ} \mathrm{kg}^{-1}{ }^{\circ} \mathrm{C}^{-1}\right)$ & $\alpha\left(\mathrm{m}^{2} \mathrm{~s}^{-1}\right)$ & $\rho\left(\mathrm{kg} \mathrm{m}^{-3}\right)$ \\
\hline Almendra fresca & 0.32 & 2.65 & $1.13 \times 10^{-7}$ & 1138.6 \\
\hline
\end{tabular}

Al comparar los valores obtenidos de la ecuación de Choi \& Okos (1985) como se observa en la tabla 4, con la herramienta DEPROTER elaborado por Alvis et al. (2012) no se observaron diferencias significativas. La difusividad y conductividad térmica, el calor especifico y la densidad de almendras frescas fue de $1.13 \times 10^{-7}$ $\mathrm{m}^{2} \mathrm{~s}^{-1}, 0,32 \mathrm{~W} \mathrm{~m}^{-1} \mathrm{C}^{-1}, 2.65 \mathrm{~kJ} \mathrm{~kg}^{-1}{ }^{\circ} \mathrm{C}^{-1}$ y $1138.6 \mathrm{~kg} \mathrm{~m}^{-3}$ respectivamente. Estos valores son similares con los obtenidos por otros autores en semillas secas; Bitra et al., (2010) reportaron en almendras (Arachis hypogaea Linnaeus) calor específico y conductividad térmica en rangos de 1.9 a $2.8 \mathrm{~kJ} \mathrm{~kg}^{-1}{ }^{\circ} \mathrm{C}^{-1}, 0.15$ a $0.19 \mathrm{~W} \mathrm{~m}^{-1} \mathrm{C}^{-1}$ respectivamente, mientras que la difusividad térmica fue de $1.1 \times 10^{-7} \mathrm{~m}^{2} \mathrm{~s}^{-1}$. Biju y Carson (2008) midieron la difusividad en cacahuete (Castanea sativa) a $30^{\circ} \mathrm{C}$ la cual fue de $5.1 \times 10^{-11} \mathrm{~m}^{2} \mathrm{~s}^{-1}$. Asimismo, se han reportado en granos de café tradicional difusividad y conductividad térmica de 0.00138 $\mathrm{cm}^{2} \mathrm{~s}^{-1}$ y $0.22 \mathrm{~W} \mathrm{~m}^{-1} \mathrm{~K}^{-1}$ y Cp de $1.64 \mathrm{~kJ} \mathrm{~kg}^{-1}{ }^{\circ} \mathrm{C}^{-1}$ (Gordillo et al., 2009). El Cp de pistachos también fue similar 2.527 a $2.707 \mathrm{~kJ} \mathrm{~kg}^{-1} \mathrm{~K}^{-1}$ para diferentes variedades (Razavi y Taghizadeh, 2007). La densidad de la almendra fue más alta que la reportada por otros autores; Demir et al. (2003) reportaron una densidad promedio de avellanas de $848 \mathrm{~kg} \mathrm{~m}^{-3}$ para almendras tostadas, sin embargo en almendras fresca reporta una densidad de $1150.6 \mathrm{~kg} \mathrm{~m}^{-3}$.

De esta manera las propiedades termofísicas de estos frutos secos varían de acuerdo al porcentaje de humedad del producto (Razavi y Taghizadeh, 2007; Bitra et al., 2010). La literatura ha revelado que muy pocas investigaciones se han llevado a cabo en almendras y ninguna en almendro de la India para determinar el calor específico, conductividad térmica y la difusividad térmica. El conocimiento de las propiedades térmicas en los granos secos, es necesaria para el modelaje, optimización y el diseño de los procesos y equipos de procesamiento en las operaciones basadas en tratamientos térmicos tales como secado, tostado, enfriamiento o congelación (Ariza y Gordillo, 2007; Bitra et al., 2010). En el caso de almendras las operaciones de secado y tostado se llevan a cabo para eliminar microorganismos y sus toxinas, así como para proporcionar una textura crujiente y obtención de aromas y sabores característicos (Demir et al., 2003).

\section{Análisis sensorial almendras tostadas}

Se realizó análisis sensorial (Figura 1) a almendras tostadas a temperaturas de 110 y $140{ }^{\circ} \mathrm{C}$; Los resultados obtenidos en la prueba de cata, muestran que las almendras tostadas a temperaturas de $1400^{\circ} \mathrm{C}$ fueron las más aceptadas por los consumidores. Observándose que el $68,7 \%$ de la población encuestada equivalente a 46 personas les gusto mucho la almendra tostada a $140{ }^{\circ} \mathrm{C}$, mientras que el $11,4 \%$ equivalente a 8 personas les gusta mucho el tratamiento de $110^{\circ} \mathrm{C}$ (Figura 1). Según los resultados arrojados en la prueba de Tukey para comparación de medias de tratamiento, se encontró que hay diferencia significativa entre las almendras tostadas a $140{ }^{\circ} \mathrm{C}$ y $110^{\circ} \mathrm{C}$, existiendo un mayor grado de satisfacción por la almendra tostada a $140^{\circ} \mathrm{C}$ por parte de los consumidores. 


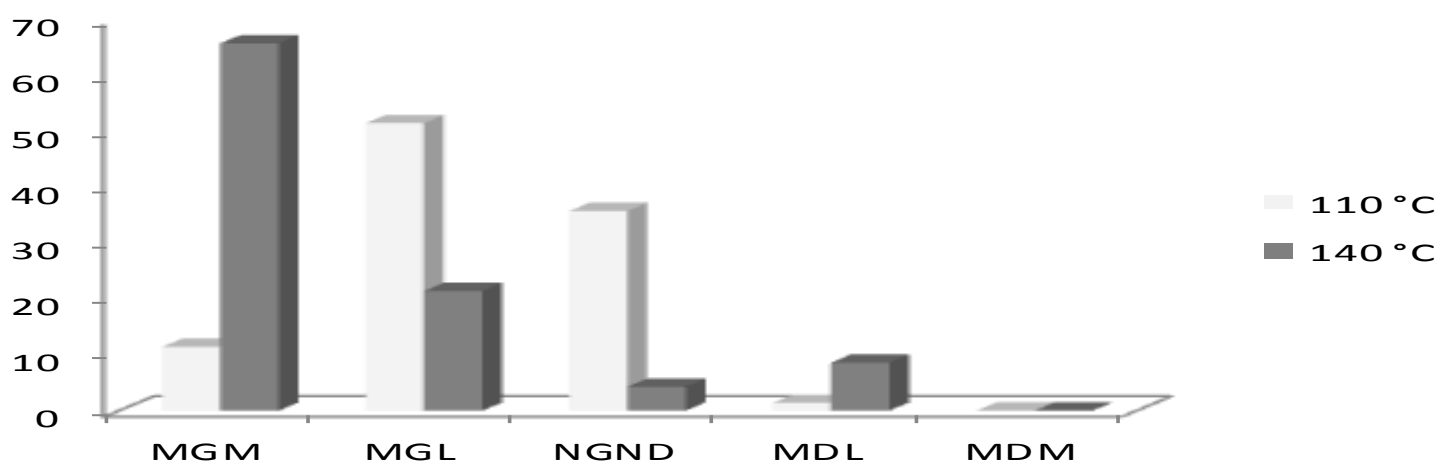

Fig. 1. Puntuaciones de la evaluación sensorial por el panel de consumidores (MGM: me gusta mucho; MGL: me gusta ligeramente; NGND: no gusta, no disgusta; MDL: me disgusta ligeramente; MDM: me disgusta mucho)

Varela et al. (2008) estudiaron el tostado de almendras (Prunus amygdalus L.), a $200{ }^{\circ} \mathrm{C}$ por $1.5,3,4.5$ y 6 minutos observando que los consumidores presentaron más aceptabilidad por las muestras de almendras más tostadas, tanto con respecto a la aceptabilidad global como en la textura final del producto. Los cambios en el color, la textura y en el sabor de almendras tostadas están directamente relacionados con la temperatura y el tiempo de tostado (Demir y Cronin, 2005; Lasekan y Abbas, 2010). Las altas temperaturas de tostado de almendras pueden causar la degradación térmica de los constituyentes naturales de la cáscara de almendras sobre todo los compuestos con carácter antioxidante, como polifenoles, no obstante esta actividad antioxidante puede aumentar durante el tostado debido a los productos de la reacción de Maillard (Bolling et al., 2010; Davis et al., 2010). El proceso de calentamiento cataliza la reacción entre aminoácidos y azúcares a través de la reacción de Maillard, que es responsable del desarrollo de color, y los compuestos volátiles heterocíclicos en las nueces tostadas (Lasekan y Abbas, 2010).

\section{CONCLUSIONES}

El contenido de grasa del fruto del almendro, es dependiente de la región geográfica donde se cultive, sin embargo, las diferencias encontradas en ceniza y humedad, requieren de posterior investigación en relación a la influencia del clima. La nuez de la almendra contiene cantidades importantes de nutrientes, similares a lo establecido para otros frutos secos como avellanas, maní y nueces, que la convierten en una alternativa nutricional, por lo que podría considerarse la posibilidad de cultivarla en forma intensiva, y destinarla a la elaboración de productos de tipo industrial como almendras confitadas, harinas, hojuelas, tostadas, entre otros. Para el aprovechamiento de estos productos en la industria alimenticia, sobre todo como almendras tostadas, es importante conocer las propiedades termofísicas, las cuales según esta investigación son muy similares a los encontrados por otros autores en diversos frutos secos.

\section{REFERENCIAS}

Alvis, A., I. Caicedo y P. Peña, Determinación de Propiedades Termofísicas de Alimentos en Función de la Concentración y la Temperatura empleando un Programa Computacional. Inf. tecnol., vol.23, no.1, p.111116. ISSN 0718-0764. (2012).

Ariza, C. H. y D. F. Gordillo, Uso de la Técnica Fotoacústica para la Caracterización de Café Proveniente del Eje Cafetero Colombiano. Rev. Acad. Colomb. Cienc., 31(119): 217-234. (2007).

Biju C. A. y K. Carson, Drying curves and apparent diffusivity of New Zealand chestnut variety '1015'. Journal of Food Engineering 85: 381-386. (2008).

Bitra, V., S. Banu, P. Ramakrishna, G. Narender y A. Womac, Moisture dependent thermal properties of peanut pods, kernels, and shells. Biosystems Engineering 106 :506-512. (2010).

Bolling, B.W., J. B. Blumberg y O. Chen, The influence of roasting, pasteurisation, and storage on the polyphenolcontent and antioxidant capacity of California almond skins. Food Chemistry, 123 (4) 15: 10401047. (2010). 
Buelvas, H., Y. Arrieta, G. Arrázola y P. Martínez, Análisis fisicoquímico y agronómico de frutos y semillas de almendro de la India (Terminalia catappa L.). Fruticultura Profesional 166: 37-44. (2007).

Chen, C.Y., y J. B. Blumberg, Phytochemical composition of nuts. Asia Pacific Journal 381 of Clinical Nutrition, 17, 329-332. (2008).

Choi Y. y M. Okos, Effects of temperature and composition on the thermal properties of foods. Food Engineering and Process Applications 1: 93-101. (1985).

Davis, J.P., L.L. Dean, K. M. Price y T. H. Sanders, Roast effects on the hydrophilic and lipophilic antioxidant capacities of peanut flours, blanched peanut seed and peanut skins. Food Chemistry 119: 539-547. (2010).

Demir, A. D., P. Baucour, K. Cronina y K. Abodayeh, Analysis of temperature variability during the thermal processing of hazelnuts. Innovative Food Science and Emerging Technologies 4: 69-84. (2003).

Demir, D.A. y K. Cronin, Modelling the kinetics of textural changes in hazelnuts during roasting. Simulation Modelling Practice and Theory 13 : 97-107. (2005).

Feyissa, A. H., Robust Modelling of Heat and Mass Transfer in Processing of Solid Foods. Tesis de Ph.D. National Food Institute, Food Production Engineering, Universidad Técnica de Dinamarca. (2011).

González, M.M., F. Mendoza, J. Mora, M. Mendoza, J. Márquez y B. Bravo, Valor nutricional de la semilla del almendrón (Terminalia catappa Linn). Revista de la facultad de farmacia 47 (1): 25 - 29. (2005).

Gordillo D. F., V. A. Ortega y R. F. Zarate, Correlación Entre Parámetros Térmicos de Café de Diferente Origen. Revista Colombiana de Física 41(1). (2009).

Hassan, H. y H. Ramaswamy, Measurement and targeting of thermophysical properties of carrot and meat based alginate particles for thermal processing applications. Journal of Food Engineering 107: 117-126. (2011).

Inbaraj, B.S. y N. Sulochana, Mercury adsorption on a carbon sorbent derived from fruit shell of Terminalia catappa. Journal of Hazardous Materials B133: 283-290. (2006).

Lasekan, O. y K. Abbas, Analysis of volatile flavour compounds and acrylamide in roasted Malaysian tropical almond (Terminalia catappa) nuts using supercritical fluid extraction. Food and Chemical Toxicology 48: 2212-2216. (2010).

Nagappa, A.N. Thakurdesai. P.A. Venkat R. N, Jiwan Singh, Antidiabetic activity of Terminalia catappa Linn fruits. Journal of Ethnopharmacology 88. 45-50. (2003).

Thomson, L.A. y B. Evans, Species Profiles for Pacific Island Agroforestry. http://www.traditionaltree.org/ [28 septiembre 2010]. (2006).

Razavi, S. M. y M. Taghizadeh, The specific heat of pistachio nuts as affected by moisture content, temperature, and variety. Journal of Food Engineering 79: 158-167. (2007).

Varela, P., A. Salvador y S. Fiszman, On the assessment of fracture in brittle foods: The case of roasted almonds. Food Research International 41: 544-551. (2008). 